\title{
Questões e modelos de avaliação e intervenção em Psicologia Escolar: o caso da Europa e América do Norte
}

\author{
Assessment and intervention issues and models in \\ School Psychology: The case of Europe \\ and North America
}

João LOPES ${ }^{1}$

Leandro da Silva ALMEIDA²

\begin{abstract}
Resumo
As práticas da Psicologia Escolar parecem ser cada vez mais marcadas pelas necessidades de referenciação/diagnóstico de crianças para o subsistema de educação especial, em detrimento do desenho e implementação de intervenções dirigidas aos problemas específicos dos alunos. A aparente insatisfação dos psicólogos escolares com essa tendência, bem como as dificuldades na utilização de modelos categoriais de diagnóstico em contexto escolar, têm dado origem à progressiva implementação de modelos alternativos de avaliação e intervenção, principalmente de modelos Response to Intervention, Curriculum-Based Measurement e Problem Solving. A controvérsia quanto à natureza verdadeiramente alternativa desses modelos parece, no entanto, longe de se esgotar. Neste artigo são discutidas vantagens e limitações dos diferentes modelos, de acordo com a melhor evidência disponível na literatura, e são ainda equacionadas as suas implicações nas práticas da Psicologia Escolar.
\end{abstract}

Palavras-chave: Avaliação; Diagnóstico; Intervenção; Distúrbios da aprendizagem; Educação especial.

\begin{abstract}
Practices in School Psychology seem to be increasingly restricted to referrals/diagnosis of children for the sub-system of special education instead of being focused on the design and implementation of interventions for students with specific problems. The apparent dissatisfaction of school psychologists with this trend and the difficulties dealing with categorical diagnostic models within the school context have stimulated a movement toward the implementation of alternative assessment and intervention models, such as Response to Intervention, Curriculum-Based Measurement and Problem-Solving. However, the controversy about the true alternative nature of these models seems far from being exhausted. The aim of this paper is to discuss the benefits and limitations of the different models according to the best evidence available. We also consider the implications for practices in School Psychology.
\end{abstract}

Keywords: Assessment; Diagnosis; Intervention; Learning disabilities; Special education.

$\boldsymbol{\nabla} \nabla \boldsymbol{v}$

1 Universidade do Minho, Escola de Psicologia. Campus de Gualtar, 4710-057, Braga, Portugal. Correspondência para/Correspondence to: J. LOPES. E-mail: <joaols@psi.uminho.pt>.

2 Universidade do Minho, Instituto de Educação. Braga, Portugal. 
A Psicologia Escolar enquanto disciplina emergiu como tentativa de compreender e lidar com problemas de comportamento e aprendizagem de crianças e adolescentes (Cole \& Siegel, 1990; Tharinger \& Lambert, 1999). No seu percurso evolutivo, mobilizou diversas disciplinas e saberes, como a Psicologia da Educação, a Psicologia do Desenvolvimento, a Psicopatologia, a Psicopatologia do Desenvolvimento etc., o que desde logo balizou muitas das práticas de avaliação e de intervenção dos psicólogos escolares (Jimerson, 2014; Reschly, 2003).

Mais recentemente, a extensão da escolaridade obrigatória colocou novos e complexos problemas, quer em termos de aprendizagens, quer em termos comportamentais. Neste último caso, importa destacar o significativo aumento da preocupação com a violência juvenil em contexto escolar (Hirschfield \& Gasper, 2011; Johnson \& Barton-Bellessa, 2014). Por outro lado, o abandono escolar, até há alguns anos tolerado (apesar do caráter obrigatório da escolaridade básica), deixou de ser aceito, assistindo-se em muitos países ao desenvolvimento de práticas, igualmente compulsivas, de manutenção no sistema de ensino de adolescentes que eventualmente desejam abandoná-lo ou nele desinvestiram (De Witte, Cabus, Thyssen, Groot, \& Van Den Brink, 2013).

O resultado mais evidente de tais políticas, no que diz respeito à Psicologia Escolar, foi o aumento muito significativo do peso da avaliação nas práticas profissionais dos psicólogos, ainda que não por vontade própria (Reschly, 2003) (Tabela 1). Em pouco mais de três décadas, o tempo dedicado pelos psicólogos escolares à avaliação e colocação de alunos num tipo qualquer de sistema especial de apoio, em muitos países da Europa e nos Estados
Unidos, terá passado de 30 a 40\% para o patamar de 70 a $80 \%$ - havendo mesmo muitos casos em que essa percentagem será superior (Ysseldyke, 2005). Desse tempo, cerca de metade será gasto em atividades de avaliação individual, normalmente com recurso a medidas estandardizadas de capacidades ou de realização (Castillo, Curtis, \& Gelley, 2012; Reschly \& Ysseldike, 1995; Vanderwood \& Burns, 2013).

Nesse contexto, a atividade da Psicologia Escolar, pelo menos nos países referidos, parece centrar-se cada vez mais na aplicação de instrumentos que se supõe serem capazes de despistar, identificar ou diagnosticar problemas ou perturbações de aprendizagem e comportamento dos alunos. Porém, dado que a avaliação está estreitamente ligada às políticas de referenciação, sobretudo para a educação especial, os psicólogos passaram a ter que evidenciar psicometricamente a indispensabilidade de integração de determinado aluno no subsistema de educação especial, isto é, tiveram que fazer cada vez mais prova da precisão dos seus diagnósticos (Kauffman, Lloyd, Hallahan, \& Astuto, 1995; Kauffman \& Lopes, 2007). Essa exigência prende-se às limitações de financiamento do subsistema e traz consequências significativas para a prática dos psicólogos escolares. Desde logo, a afetação de cada vez mais tempo à avaliação faz diminuir o tempo dedicado à intervenção, promove o recurso sistemático a instrumentos de avaliação normativos, proporciona uma transformação conceptual de variáveis contínuas (e.g. a competência leitora) em variáveis categoriais (diagnósticos) e favorece o recurso a modelos de tipo AptitudeTreatment Interaction (ATI, Interação Aptidão-Tratamento), entre outros (Brown-Chidsey \& Steege, 2010; Christ, Burn, \& Ysseldyke, 2005; Gresham, 2007; Ysseldyke, 2005).

Tabela 1

Estimativa do tipo de trabalho desenvolvido semanalmente pelos psicólogos escolares (Rechly, 2003)

\begin{tabular}{lcc}
\hline Tipo de trabalho & Horas desejadas por semana & Horas efetivas por semana \\
\hline Avaliação psicoeducacional & 13 & 23 \\
Intervenções diretas & 12 & 7 \\
Consulta para resolução de problemas & 6 & 9 \\
Consulta organizacional-sistêmica & 3 & 4 \\
Intervenção-avaliação & 2 & 3 \\
\hline
\end{tabular}




\section{Apoios educativos e modelos de avaliação/intervenção}

A prática psicológica sistematicamente orientada para o diagnóstico merece ser contextualizada, uma vez que não pode ser entendida como mero capricho dos psicólogos envolvidos (Vanderwood \& Burns, 2013). Na verdade, obedece a parâmetros bem definidos e tem suas raízes na necessidade de dar resposta a alunos que, por via do alargamento progressivo da escolaridade obrigatória, foram permanecendo cada vez mais tempo na escola, apesar da sua dificuldade em alcançar níveis aceitáveis de sucesso (Lopes, 2010). O desenvolvimento relativamente recente de políticas de inclusão de todos os alunos nas salas de aula regulares, independentemente de sua condição ou deficiência, contribuiu também para o significativo alargamento dos serviços de identificação/diagnóstico (Kauffman et al., 1995).

A criação de serviços categoriais de identificação de alunos com problemas (i.e. enquadrar tais alunos numa categoria específica de diagnóstico e de apoio/terapia) não decorre exclusivamente dos avanços científicos na área. Depende, em larga medida, da limitação do número de sujeitos elegíveis em função dos recursos materiais e humanos disponíveis, significando que a avaliação deve apoiar a decisão de quais os alunos com necessidades que irão receber apoio, e não propriamente identificar aqueles com necessidade de apoio (Ysseldyke \& Marston, 1998). Teoricamente, isso implica um sistema que seja capaz de, numa primeira fase, separar de forma válida e fiável os sujeitos que precisam de apoio (verdadeiros positivos) dos que não precisam de apoio (negativos), e, numa segunda fase, separar os positivos (que apresentam o problema) elegíveis dos positivos não-elegíveis. Tal sistema, pelas fragilidades intrínsecas, é contestado na validade e fiabilidade de suas categorias, assim como de sua real eficiência (Fletcher, Denton, \& Francis, 2005; Kearns \& Fuchs, 2013; Sanches-Ferreira, Silveira-Maia, \& Alves, 2014; Reschly \& Gresham, 1989; Ysseldyke, 2005; Ysseldyke \& Marston, 1998).

A concepção categorial dos problemas escolares radica nos clássicos modelos ATI (Cronbach,
1957), cuja ideia fundamental é a de que, para um determinado tipo de pessoa que apresenta um determinado tipo de problema, existirá uma intervenção/tratamento muito específico. Todavia, como referem Reschly e Ysseldyke (1995), apesar de essa ideia ser intuitivamente atrativa e de parecer que deveria funcionar, não existe evidência suficiente que suporte a sua validade enquanto metodologia de psicologia aplicada. O próprio Cronbach viria a abandonar essa ideia (Cronbach, 1975) por considerar que todos os esforços por ele desenvolvidos para validar o modelo não revelaram mais do que efeitos inconsistentes ou demasiado tênues.

Ainda que com variações consideráveis de país para país, os modelos de tipo ATI têm modelado as práticas da Psicologia Escolar (Burns \& Ysseldyke, 2009), satisfazendo as necessidades de referenciação dos sistemas escolares e sociais de apoio, apesar de muitas das intervenções daí derivadas carecerem de validade. Na verdade, e a título meramente ilustrativo, programas de treino psicomotor para dificuldades da leitura ou métodos de ensino baseados em estilos de aprendizagem são hoje generalizadamente considerados ineficazes (Fletcher et al., 2005; Swanson, Harris, \& Graham, 2013).

No contexto escolar, a explicação para os problemas emocionais, comportamentais e, sobretudo, de aprendizagem, bem como seu diagnóstico através de instrumentos de validade psicométrica confirmada, assentou desde sempre na noção de que o fracasso, déficit ou perturbação, são intrínsecos ao sujeito (within-subject). Eles traduzem traços mais ou menos estáveis que deverão ser determinados e avaliados através da utilização de testes referenciados à norma (Spear-Swerling \& Sternberg, 1996; 1998).

Nesse contexto, o papel da Psicologia foi-se orientando progressivamente para diagnóstico dos problemas de aprendizagem e comportamento dos alunos, acompanhado de uma sugestão de encaminhamento ou terapia. Contudo, o aparentemente imparável aumento do número e diversidade dos problemas tem obrigado à criação de sucessivas categorias, como por exemplo as Emotional and Behavioral Disorders (EBD, Perturbações Emocionais e Comportamentais), perturbações da fala e do 
discurso, desvantagem social, disfuncionalidade familiar, bilinguismo e, sobretudo, as dificuldades de aprendizagem, categoria em que mais sujeitos são incluídos (cerca de 50\%) (Ysseldyke \& Marston, 1998).

Essa proliferação de categorias não resolve eficazmente o complexo problema da identificação/ diagnóstico e menos ainda o da intervenção (Fletcher, Lyon, Fuchs, \& Barnes, 2007). No caso das Dificuldades de Aprendizagem (DA), por exemplo, a avaliação deve decidir dicotomicamente ("tem" ou "não tem" DA) na base de variáveis que têm uma distribuição contínua (e.g. a leitura, a escrita ou o cálculo), sem nenhum ponto de corte evidente (Fletcher et al., 2007). Tal procedimento, assente em categorias de validade e fiabilidade duvidosas, para além do tempo e recursos que consome, deixa os avaliadores vulneráveis perante processos de contestação que, em alguns países, são dirimidos em tribunal (e.g. Larry P. versus Riles, 1979/1986). Por outro lado, o esforço e recursos investidos na categorização parecem ter um impacto muito relativo na intervenção. Alguns autores sugerem que o tipo de intervenção praticamente não varia se o aluno apresenta dificuldade de aprendizagem, deficiência mental ligeira ou qualquer outro tipo de déficit acadêmico no âmbito da leitura, escrita e cálculo (Reschly \& Ysseldyke, 1995; Ysseldyke, Algozzine, \& Epps, 1983). Assim sendo, a contestação crescente às classificações categoriais, incluindo contestações judiciais, sugere que, apesar de os testes de referência à norma apresentarem características psicométricas cada da vez mais sólidas, a refutação dos seus resultados e conclusões por parte dos pais ou encarregados de educação recebe acolhimento em diversas instâncias escolares e/ou judiciais (Burns, Deno, \& Jimerson, 2007; Christ et al., 2005).

Duas falácias nessa avaliação diagnóstica merecem ser consideradas. A primeira tem a ver com a questionável validade de alguns instrumentos psicométricos para avaliar certos problemas educacionais, enquanto a segunda prende-se à crença errônea de que da aplicação de tais instrumentos decorrem necessariamente boas indicações para a 78 intervenção. A utilização de testes de QI, que cons- titui a forma mais comum de avaliar a discrepância entre capacidade cognitiva e realização acadêmica (Fletcher, Morris, \& Lyon, 2003; Peterson \& Shinn, 2002), é o exemplo mais evidente de testes com boas qualidades psicométricas que não avaliam diretamente o problema e, sobretudo, que fornecem muito poucas indicações úteis para a intervenção (Barnett, Daly, Jones, \& Lentz, 2004; Case, Speece, \& Molloy, 2003; Ortiz, 2004).

\section{Modelos não categoriais de avaliação/intervenção}

A insatisfação com os resultados da Psicologia Escolar não é recente (Burns \& Ysseldyke, 2009; Ysseldyke, 1973), originando o desenvolvimento de modelos de avaliação e intervenção mais centrados nos problemas dos alunos do que nas necessidades de referenciação, como é o caso dos modelos de Resolução de Problemas (Deno, 2008), dos modelos Response to Intervention (RTI, Resposta à Intervenção) (Gresham, 2007), dos modelos de Avaliação por Referência ao Currículo (Deno, 1989; 2003) e da Análise Funcional do Comportamento (Steege \& Watson, 2009).

A defesa de uma mudança de paradigma - dos modelos correlacionais para os modelos experimentais de resolução de problemas -, também não é recente (Fuchs, Mock, Morgan, \& Young, 2003). O próprio Cronbach, em 1975, sugeriu que a alternativa aos modelos ATI seria a avaliação contextual dos problemas e a monitorização da intervenção, tendo em vista seu eventual ajustamento, ou seja, um modelo muito próximo dos atuais modelos de resolução de problemas (Reschly \& Ysseldyke, 1995).

Alguns anos mais tarde, num documento intitulado "School psychology: A blueprint for training and practice", Ysseldyke, Reynolds e Weinberg (1984) sugeriram a eliminação da classificação categorial dos alunos, substituindo-a por avaliações e intervenções realizadas nos contextos em que os problemas ocorrem, através de medidas comportamentais e de Curriculum-Based Measurement (CBM, Medidas Baseadas no Currículo), no quadro de um modelo de resolução de problemas. Em tal 
contexto, o papel dos psicólogos deixaria de ser o de diagóstico/classificação, focalizando-se antes no apoio à instrução na sala de aula, ou seja, numa estratégia de consultoria para a resolução de problemas.

Numa perspectiva de Problem Solving (PS, Resolução de Problemas) considera-se que um problema existe sempre que é percebida uma discrepância entre o que o sujeito deveria realizar e aquilo que ele efetivamente realiza (Deno, 2008). A formulação parece relativizar a discussão sobre se o sujeito tem ou não tem o problema, mantendo a relevância da avaliação psicológica, da qualidade dos instrumentos para tal avaliação e da validade das intervenções (Deno, 1985; Fuchs et al., 2003; Lopes, Rutherford, Cruz, Mathur, \& Quinn, 2011).

A insatisfação provocada pela categorização dos alunos associa-se à adoção de modelos RTI, apesar de dificuldades inerentes a imprecisões ou indefinições terminológicas junto dos utilizadores (Burns et al., 2007; Christ et al., 2005). Por exemplo, no que diz respeito às relações e/ou diferenças entre RTI, PS e CBM, Burns et al. (2007) sugerem que o termo PS se aplica a um processo sistemático cujo objetivo é mudar os resultados dos alunos, enquanto a RTI constitui forma sistemática de determinar se a mudança ocorreu e em que circunstâncias isso se deu. Já o CBM refere-se a um processo de mensuração que tem por referência o currículo com o qual o desempenho do sujeito é contrastado, tendo em vista o ajustamento da instrução. Isso explica, aliás, tratar-se de um modelo originalmente desenvolvido para testar a eficácia das intervenções na área da educação especial (Deno, 1985; 2003). Para além das diferenças terminológicas, é possível supor uma diferença importante entre esses modelos: enquanto os modelos CBM e PS não implicam um diagnóstico formal do problema, os modelos RTI preveem a hipótese de diagnóstico no caso de o sujeito não responder positivamente à intervenção (Fletcher et al., 2003).

Fletcher et al. (2007) sublinham que os modelos RTI não representam uma alternativa radical aos modelos categoriais de classificação das dificuldades de aprendizagem, uma vez que implicam sempre uma tomada de decisão dicotômica relati- vamente aos sujeitos avaliados, ou seja, um problema classificatório. No quadro dos próprios modelos RTI é possível considerar que o " $R$ " significa "resposta" ou "resistência" (Christ et al., 2005). No primeiro caso, em que todos os alunos são testados independentemente de qualquer suspeita de problema, as decisões sobre a instrução, frequentemente sob a forma de alocação de recursos, antecedem qualquer preocupação diagnóstica (trata-se de um tipo de modelo filiado na análise aplicada de comportamento, que procura explicações funcionais e não explicações estruturais para o problema). No segundo caso, a implementação da intervenção visa testar a resistência do sujeito às estratégias utilizadas, sendo a elegibilidade para a educação especial, ou seja, a classificação, uma preocupação central (Gresham, 2006). Do mesmo modo, pode conceber-se que interpretar o "I" (de RTI) como instrução traduz uma perspectiva não classificatória do modelo, enquanto interpretá-lo como intervenção remete para um tipo de adaptação instrucional intensiva e altamente modificada, típica da educação especial (Barnett et al., 2004; Case et al., 2003).

No quadro desses modelos, independentemente de seu formato específico, são questões de maior relevo: os tipos de medida a utilizar; a frequência da avaliação; a validade, fiabilidade, sensibilidade e usabilidade das medidas; a ligação das medidas à intervenção e ao follow-up, entre outras. Brown-Chidsey (2008), Ortiz (2004) e Salvia e Ysseldyke (2003) salientam que os testes de referência à norma, tipicamente utilizados para determinar discrepâncias entre capacidades cognitivas e realização acadêmica, são utilizados em muitos casos com sujeitos cujo background é demasiado diferente do da amostra normativa para que sejam utilizados de forma fiável com esses sujeitos. Sugerem ainda que esses testes têm em geral pouca utilidade instrucional e que a comparação de resultados de baterias de testes para determinar as discrepâncias é problemática, uma vez que as normas desenvolvidas para os diferentes testes não são necessariamente comparáveis (possibilidade de serem superadas pela estimação das capacidades e/ou conhecimentos no quadro da Teoria de Resposta ao Item). 
O processo de condução de um modelo RTI (níveis de avaliação, níveis de instrução etc.), numa escola, com avaliações referenciadas ao currículo, obedece a especificidades que é necessário considerar (Tabela 2).

O modelo sugere a necessidade de as avaliações serem frequentes (pelo menos três avaliações anuais dos alunos envolvidos), com recurso a testes referenciados ao currículo (CBM), havendo preocupação com a qualidade psicométrica de tais instrumentos. Essas avaliações, para além de aferirem o estado inicial do sujeito, são concebidas para estimar a eficácia do programa de intervenção subsequente à avaliação inicial (e que pode ser simplesmente o ensino na sala regular) (Barnett et al., 2004; Gresham, 2007; Griffiths, Parson, Burns, VanderHeyden, \& Tilly, 2007).

Da avaliação de nível mais elementar, destinada a todos os alunos, resulta uma decisão: não fazer nenhum tipo de intervenção porque o ensino parece revelar-se satisfatório, ou fazer uma intervenção. Neste último caso, uma segunda decisão deverá ser tomada: intervir sobre a turma inteira (por fraco rendimento ou comportamento coletivo, por exemplo), em geral sobre a forma como o ensino está sendo ministrado, ou intervir sobre alunos específicos (Jimenez, 2010). Neste segundo caso, os alunos poderão, mediante o juízo que se fizer relativamente ao montante de apoio disponível (e.g. em termos de recursos humanos, materiais, extensão temporal do apoio), ser referenciados para um apoio relativamente limitado (Nível II) ou para um apoio extensivo (Nível III). Tendo em conta os valores esperados relativamente a cada um desses níveis (1-5\% de alunos no Nível III, 5-10\% no Nível II, e $80-90 \%$ no Nível I), qualquer desvio significativo (por exemplo, 40\% dos alunos serem referenciados para o Nível II) poderá significar mais um problema de ensino, inclusive do professor, do que um problema de ensino-aprendizagem de alunos específicos (Justice, 2006; Koutsoftas, Harmon, \& Gray, 2009).

A detecção de alunos com problemas específicos, que necessitam de apoio e instrução suplementar à fornecida pelo professor na sala de aula, configura a sinalização de um problema. O fato de o problema ser percebido e de o modelo prever a adoção imediata de medidas conducentes à sua redução ou eliminação, evidencia que os modelos RTI seguem uma lógica muito parecida com a dos modelos PS. No quadro destes últimos, basta que seja percebida uma discrepância entre o que é esperado e aquilo que o aluno efetivamente realiza para que se considere que o problema existe. A essa altura, não interessa prolongar a discussão sobre se o problema existe, tão comum nas referenciações para os sistemas de educação especial, mas apreciar a grandeza da discrepância encontrada (Deno, 2003; Fuchs \& Deno, 1991).

A diferença entre os modelos RTI e os modelos PS torna-se porventura mais saliente na especificação dos critérios de elegibilidade para apoio (seja de Nível II, seja de Nível III). Enquanto nos modelos RTI a elegibilidade resulta da "resistência" do sujeito à intervenção (a qual é acionada depois

\section{Tabela 2}

Avaliação/intervenção acadêmica e/ou comportamental no quadro de um modelo RTI/CBM

\begin{tabular}{|c|c|c|c|}
\hline & Rendimento acadêmico & Percentagem de alunos abrangidos & Comportamento \\
\hline 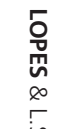 & $\begin{array}{l}\text { Nível III: Intervenção intensiva apenas para alunos } \\
\text { (muito poucos) que precisam de instrução intensiva. }\end{array}$ & $1-5$ & $\begin{array}{l}\text { Nível III: Intervenção intensiva apenas para alunos } \\
\text { (muito poucos) que precisam de intervenções indi- } \\
\text { viduais. }\end{array}$ \\
\hline $\begin{array}{l}n \\
\frac{2}{3} \\
\frac{m}{3}\end{array}$ & $\begin{array}{l}\text { Nível II: Intervenção dirigida a grupos específicos que } \\
\text { precisam de mais algum apoio, para além da instru- } \\
\text { ção normal. }\end{array}$ & 5-15 & $\begin{array}{l}\text { Nível II: Intervenção dirigida a grupos específicos } \\
\text { que precisam de alguma monitorização comporta- } \\
\text { mental específica. }\end{array}$ \\
\hline & $\begin{array}{l}\text { Nível I: Intervenções universais (ensino regular para } \\
\text { todos os alunos). }\end{array}$ & $80-90$ & $\begin{array}{l}\text { Nível I: Intervenções universais (gestão de sala de } \\
\text { aula dirigida a todos os alunos). }\end{array}$ \\
\hline
\end{tabular}

Nota: RTI: Response To Intervention; CBM: Curriculum-Based Measurement. 
de detectado o problema na avaliação inicial), nos modelos PS a percepção da existência de um problema é suficiente para que o sujeito receba apoio extra, sem preocupação específica com a classificação ou elegibilidade do problema. Desse modo, enquanto nos primeiros a tomada de decisão resulta de duas ou mais avaliações consecutivas, nos modelos PS a tomada de decisão resulta da apreciação que o professor faz diariamente do desempenho acadêmico e comportamental do aluno ("problems exist in the eye of the beholder") (Deno, 2008, p.10).

\section{Limitações dos novos modelos de avaliação}

Os modelos mais recentes de avaliação/intervenção escolar pretendem entre outras coisas detectar com diligência os problemas de ensino e aprendizagem, fornecer indicações precisas sobre formas de intervenção oportunas e eficazes, e libertar professores e psicólogos do peso dos procedimentos de referenciação para os apoios educativos (Fletcher \& Vaughn, 2009; Griffiths et al., 2007; Jackson, Pretti-Frontczak, Harjusola-Webb, Grisham-Brown, \& Romani, 2009; Jimenez, 2010; Jimenez et al., 2010; Vaughn \& Fletcher, 2010).

Contudo, alguns autores (Reynolds \& Shaywitz, 2009a, 2009b) têm apresentado críticas e apontado limitações significativas de alguns desses modelos, em particular dos RTI. Os autores acima citados consideram francamente otimistas o entusiasmo dos proponentes dos modelos RTI com a eficácia dos modelos, facilidade de implementação, extensão de aplicação nas escolas, clareza quanto ao que é responsiveness (o "R" de RTI) e capacidade de resposta aos problemas dos alunos, em particular aos de aprendizagem. Reynolds e Shaywitz (2009a) questionam mesmo se se está perante um verdadeiro modelo de entrega de serviços ou perante mais um modismo educacional. Também Swanson (2008) encontra effect sizes equívocos no que se refere à eficácia dos modelos RTI. Outros autores como Kavale, Kauffman, Bachmeir e LeFever (2008) consideram que não existe evidência suficiente para suportar a superior eficácia dos modelos RTI, e que estes constituem basicamente um fenômeno sociopolítico.

Os modelos RTI têm também sido criticados por não diminuírem a carga avaliativa e de trabalho dos professores, antes aumentando-a significativamente. Do mesmo modo, é contestada a ideia da aplicação universal do modelo sem um suporte empírico forte. Nesse particular, Reynolds e Shaywitz (2009a) sustentam que aquilo que funciona localmente não funciona necessariamente bem em nível geral, ao contrário do que afirmam os defensores do modelo. Os críticos consideram ainda que, no caso específico das dificuldades de aprendizagem (de longe o grupo mais referenciado em contexto escolar), os modelos RTI podem estar substituindo o tradicional wait for fail model por um watch them fail model, sem proveito algum para os alunos em dificuldades e, ainda, com custos significativos para o sistema educativo.

VanDerHayden e Burns (2013) consideram que a avaliação e despiste universal constituem um esforço que muitos sistemas implementam de forma ineficiente e com erros. Embora o método possa operar relativamente bem em sistemas em que a maior parte dos alunos funciona acima de um patamar estabelecido de risco, no caso contrário o esforço é propenso a erros, uma vez que mesmo alunos com dificuldades poderão ser considerados como razoáveis. VanDerHayden e Witt (2005), num estudo sobre a eficácia diferencial do despiste de alunos em dificuldades, já tinham evidenciado que a referenciação pelos professores é menos fiável do que a referenciação pelo método RTI, mas que ambos os métodos são mais fiáveis em classes de nível médio do que em classes com níveis mais baixos, o que levanta dúvidas sobre as circunstâncias em que os modelos RTI são verdadeiramente fiáveis.

Moats, Kukik e Pasternack (2010), embora reconhecendo a relevância de algumas críticas, sustentam, em defesa dos modelos RTI, que muitos anos de investigação evidenciam que: (a) os alunos em risco de fracasso na leitura (os mais numerosos de entre os que precisam de ajuda) podem ser identificados nas escolas através de procedimentos baratos e desburocratizados; (b) a intervenção é mais eficaz e menos dispendiosa no início da escola- 
ridade; (c) uma intervenção precoce, fora do sistema de educação especial, pode ser suficiente para ajudar muitas crianças com potenciais problemas de aprendizagem; e (d) a sala de aula regular e a instrução suplementar são fundamentais para melhorar os resultados desses alunos. Além disso, referem que, nos Estados Unidos, antes da reforma de 2004, as escolas eram recompensadas por reprovar os alunos em vez de os ensinar, por atrasar a identificação das dificuldades, e por gastar os recursos na testagem e não na instrução. Além disso, quando colocados na educação especial, após o $3^{\circ}$ ano, esses alunos raramente se recuperavam, o que configurava, segundo os autores, um desperdício de recursos.

\section{Conclusão}

Os psicólogos e, por extensão, os professores e as escolas parecem gastar cada vez mais tempo e recursos em processos de referenciação dos alunos para sistemas de apoio ou de educação especial. Por isso, não surpreende que uma parte significativa da discussão acerca dos melhores modelos de avaliação/intervenção em contexto escolar/educacional se organize em torno desses processos de referenciação/classificação. Apesar disso, para muitos alunos a detecção dos problemas surge tardiamente, e a intervenção é ainda mais tardia, podendo em muitos casos nunca se concretizar. Nesse contexto, e atendendo a que os recursos tendem a escassear e que a responsabilização de técnicos e escolas pelos resultados tende a aumentar, dificilmente será sustentável a tradicional abordagem de "esperar para ver" o resultado das dificuldades.

Os modelos Response to Intervention, Curriculum-Based Measurement, Problem Solving apresentam-se como alternativa aos modelos de testagem por referência à norma que têm sustentado as práticas de referenciação dos sistemas de educação especial. Apesar das limitações, esses modelos parecem ter a vantagem de se organizar em torno de intervenções oportunas, descentrando-se dos modelos classificatórios de origem médica, mais focalizados na classificação dos indivíduos do

82 que na intervenção. Desse modo, parece vantajoso que a Psicologia Escolar desenvolva as suas práticas em torno de avaliações que sejam significativas para alunos e professores, e que sejam suficientemente específicas para orientar a estes últimos sobre práticas pedagógicas e comportamentos de gestão da sala de aula que permitam alcançar com eficácia os resultados desejados. Esta será eventualmente uma forma importante de evitar a irrelevância ou a desconsideração da Psicologia nas escolas, fato preocupante já assinalado há bastante tempo por alguns autores, mas para o qual essa ciência parece não ter ainda encontrado o melhor antídoto.

\section{Referências}

Barnett, D. W., Daly, E. J., Jones, K. M., \& Lentz, F. E. (2004). Response to intervention: Empirically based special service decisions from single-case designs of increasing and decreasing intensity. The Journal of Special Education, 388, 66-79.

Brown-Chidsey, R. (2008). The role of published-norm reference tests in problem-solving based assessment. In R. Brown-Chidsey (Ed.), Assessment for intervention: A problem-solving approach (pp.247-264). New York: Guilford.

Brown-Chidsey, R., \& Steege, M. W. (2010). Response to intervention: Principles and strategies for effective practice ( $2^{\text {nd }}$ ed.). New York: Guilford Press.

Burns, M. K., Deno, S. L., \& Jimerson, S. R. (2007). Toward a unified Response-to-Intervention model. In R. J. Shane, K. B. Matthew, \& A. M. VanDerHeyden (Eds.), Handbook of response to intervention: The science and practice of assessment and intervention (Vol. 4, pp.428-440). New York: Springer.

Burns, M. K., \& Ysseldyke, J. E. (2009). Prevalence of evidence-based practices in special education. Journal of Special Education, 43(1), 3-11.

Case, L. P., Speece, D. L., \& Molloy, D. E. (2003). The validity of a response-to-instruction paradigm to identify reading disabilities: A longitudinal analysis of individual differences and contextual factors. School Psychology Review, 32, 557-582.

Castillo, J. M., Curtis, M. J., \& Gelley, C. (2012). Professional practice school psychology 2010: Part 2, School psychologists' professional practices and implications for the field. Communiqué, 40, 4-6.

Christ, T. J., Burns, M. K., \& Ysseldyke, J. E. (2005). Conceptual confusion within Response-to-Intervention vernacular: Clarifying meaningful differences. Comuniqué, 34(3), 1-8.

Cole, E., \& Siegel, J. A. (1990). Effective consultation in school psychology. Toronto: Hogrefe \& Huber Publishers. 
Cronbach, L. J. (1957). The two disciplines of scientific psychology. American Psychologist, 12, 671-684.

Cronbach, L. J. (1975). Beyond the two disciplines of scientific psychology. American Psychologist, 30, 116-127.

De Witte, K., Cabus, S., Thyssen, G., Groot, W., \& Van Den Brink, H. M. (2013). A critical review of the literature on school dropout. Educational Research Review, 10, 13-28.

Deno, S. L. (1985). Curriculum-based measurement: The emerging alternative. Excepcional Children, 52, 219-232.

Deno, S. L. (1989). Curriculum-based measurement and alternative special education services: A fundamental and direct relationship. In M. R. Shinn (Ed.), Curriculum-based measurement: Assessing special children (pp.1-17). New York: Guilford Press.

Deno, S. L. (2003). Developments in curriculum-based measurement. The Journal of Special Education, 37(3), 184-192.

Deno, S. L. (2008). Problem-solving assessment. In R. Brown-Chidsey (Ed.), Assessment for intervention: A problem-solving approach (pp.10-40). New York: Guilford.

Fletcher, J. M., Denton, C., \& Francis, D. J. (2005). Validity of alternative approaches for the identification of LD: Operationalizing unexpected underachievement. Journal of Learning Disabilities, 38, 545-552.

Fletcher, J. M., Lyon, G. R., Fuchs, L. S., \& Barnes, M. A. (2007). Learning disabilities: From identification to intervention. New York: Guilford Press.

Fletcher, J. M., Morris, R. D., \& Lyon, G. R. (2003). Classification and definition of learning disabilities: An integrative perspective. In H. L. Swanson, K. R. Harris, \& S. Graham (Eds.), Handbook of learning disabilities (pp.30-56). New York: Guilford.

Fletcher, J. M., \& Vaughn, S. (2009). Response to Intervention: Preventing and remediating academic difficulties. Child Development Perspectives, 3(1), 30-37. http://dx.doi. org/10.1111/j.1750-8606.2008.00072.x

Fuchs, L. S., \& Deno, S. L. (1991). Paradigmatic distinctions between instructionally relevant measurement models. Exceptional Children, 58, 232-243.

Fuchs, D., Mock, D., Morgan, P. L., \& Young, C. L. (2003). Responsiveness-to-intervention: Definitions, evidence, and implications for the learning disabilities construct. Learning Disabilities Research \& Practice, 18(157-171).

Gresham, F. (2007). Evolution of the response-tointervention concept: Empirical foundations and recent developments. In S. R. Jimerson, M. K. Burns, \& A. M. VanDerHayden (Eds.), Handbook of response-tointervention: The science and practice of assessment and intervention (pp.10-24). New York: Springer.

Gresham, F. M. (2006). Evolution of the response-tointervention concept: Empirical foundations and recent developments. In S. R. Jimerson, M. K. Burns, \& A. M. VanDerHeyden (Eds.), Handbook of response to intervention: The science and practice of assessment and intervention (pp.10-24). New York: Springer.

Griffiths, A. M., Parson, L. B., Burns, M. K., VanderHeyden, A., \& Tilly, W. D. (2007). Response to intervention. Research for practice. Alexandria: National Association of State Directors of Special Education.

Hirschfield, P. J., \& Gasper, J. (2011). The relationship between school engagement and delinquency in late childhood and early adolescence. Journal of Youth and Adolescence, 40(1), 3-22.

Jackson, S., Pretti-Frontczak, K., Harjusola-Webb, S., Grisham-Brown, J., \& Romani, J. M. (2009). Response to intervention: Implications for early childhood professionals. Language, Speech and Hearing Services in School, 40(4), 424-434. http://dx. doi.org/10.1044/ 0161-1461(2009/08-0027)

Jimenez, J. E. (2010). Response to Intervention (RTI) Model: A promising alternative for identifying students with learning disabilities? Psicothema, 22(4), 932-934.

Jimenez, J. E., Rodriguez, C., Crespo, P., Gonzalez, D., Artiles, C., \& Alfonso, M. (2010). Implementation of Response to Intervention (RTI) Model in Spain: An example of a collaboration between Canarian universities and the department of education of the Canary Islands. Psicothema, 22(4), 935-942.

Jimerson, S. R. (2014). Enhancing science, practice, and policy relevant to school psychology around the world. School Psychology Quarterly, 29(1), 1-6.

Johnson, B. R., \& Barton-Bellessa, S. M. (2014). Consequences of school violence: Personal coping and protection measures by school personnel in their personal lives. Deviant Behavior, 35(7), 513-533.

Justice, L. M. (2006). Evidence-based practice, response to intervention, and the prevention of reading difficulties. Language, Speech \& Hearing Services in Schools, 37(4), 284-297. http://dx.doi.org/10.1044/ 0161-1461(2006/033)

Kauffman, J. M., Lloyd, J. W., Hallahan, D. P., \& Astuto, T. A. (1995). Issues in educational placement. New Jersey: Erlbaum.

Kauffman, J. M., \& Lopes, J. A. (2007). Pode a educação especial deixar de ser especial? Braga: Psiquilíbrios.

Kavale, K., Kauffman, J., Bachmeir, R., \& LeFever, G. (2008). Response-to-Intervention: Separating the rhetoric of self-congratulation from the reality of specific learning disability identification. Learning Disability Quarterly, 31, 135-150.

Kearns, D., \& Fuchs, D. (2013). Does cognitively focused instruction improve the academic performance of low-achieving students? Exceptional Children, 79(3), 263-290.

Koutsoftas, A. D., Harmon, M. T., \& Gray, S. (2009). The effect of Tier 2 intervention for phonemic awareness 
in a response-to-intervention model in low-income preschool classrooms. Language, Speech, and Hearing Services in Schools, 40(2), 116-130. http://dx.doi.org/ 10.1044/0161-1461(2008/07-0101)

Lopes, J. A. (2010). Conceptualização, avaliação e intervenção nas dificuldades de aprendizagem: A sofisticada arquitectura de um equívoco. Braga: Psiquilíbrios.

Lopes, J. A., Rutherford, R., Cruz, M. C., Mathur, S., \& Quinn, M. (2011). Competências sociais: aspectos emocionais, comportamentais e de aprendizagem ( $2^{\text {a }}$ ed.). Braga: Psiquilíbrios.

Moats, L., Kukik, S., \& Pasternack, R. (2010). Response to Reynolds and Shaywitz: Let's not go back to the Good Old Days before RTI. RTI Action Network. Retrieved June 5, 2014, from http://www.rtinetwork. org/learn/ld/response-to-reynolds-and-shaywitz

Ortiz, S. O. (2004). Nondiscriminatory assessment in schools. In C. Spielberger (Ed.), Encyclopedia of Applied Psychology (Vol. 10, pp.669-675). San Diego, C.A: Academic Press.

Peterson, K. M., \& Shinn, M. R. (2002). Severe discrepancy models: Which best explains school identification practices for learninf disbilities: A longitudinal analysis of individual differences and context factors. School Psychology Review, 31, 459-476.

Reschly, D. J. (2003). School Psychology. In W. M. Reynolds \& E. M. Gloria (Eds.), Hanbook of psychology: educational psychology (Vol. 7, pp.431-453). New York: Wiley.

Reschly, D. J., \& Gresham, F. M. (1989). Current neuropsychological diagnosis of learning problems: A leap of faith. In C. R. Reynolds (Ed.), Child neuropsychology: Techniques of diagnosis and treatment (pp.503-519). New York: Plenum.

Reschly, D. J., \& Ysseldyke, J. (1995). School psychology paradigm shift. In A. Thomas, \& J. Grimes (Eds.), Best practices in school psychology (pp.17-32). Washington, D.C: NASP.

Reynolds, C. R., \& Shaywitz, S. E. (2009a). Response to Intervention: Prevention and remediation, perhaps. Diagnosis, no. Child Development Perspectives, 3(1), 44. http://dx.doi.org/10.1111/j.1750-8606.2008.0 0075.x

Reynolds, C. R., \& Shaywitz, S. E. (2009b). Response to Intervention: Ready or not? Or, from wait-to-fail to watch-them-fail. School Psychology Quarterly, 24(2), 130-145. http://dx.doi.org/10.1037/a0016158

Salvia, J., \& Ysseldyke, J. (2003). Assessment: In special and inclusive education ( $9^{\text {th }}$ ed.). New York: Houghton Mifflin.

Sanches-Ferreira, M., Silveira-Maia, M., \& Alves, S. (2014).
Functioning, Disability and Health, version for Children and Youth (ICF-CY), in Portuguese special education assessment and eligibility procedures: The professionals' perceptions. European Journal of Special Needs Education. http://dx.doi.org/10.1080/08856257.2 014.908025

Spear-Swerling, L., \& Sternberg, R. J. (1996). Off track: When poor readers become learning disabled. Boulder: Westview Press.

Spear-Swerling, L., \& Sternberg, R. J. (1998). Curing our 'epidemic' of learning disabilities. Phi Delta Kappan, 79(5), 397-401.

Steege, M. W., \& Watson, T. S. (2009). Conducting schoo/based functional behavioral assessments. New York: Guilford.

Swanson, H. L. (2008). Neuroscience and response to instruction (RTI): A complementary role. In E. FletcherJanzen, \& C. R. Reynolds (Eds.), Neuropsychological perspectives on learning disabilities in the era of RTI: Recommendations of diagnosis and intervention (pp.28-53.). New York: Wiley.

Swanson, H. L., Harris, K. R., \& Graham, S. (2013). Handbook of learning disabilities ( $2^{\text {nd }}$ ed.). New York: Guilford.

Tharinger, D. J., \& Lambert, N. M. (1999). The apllication of developmental psychology to school psychology practice: Informing assessment, intervention, and prevention effects. In C. R. Reynolds, \& T. B. Gutkin (Eds.), School-based consultation. Theory and practice: The art and science of indirect service delivery ( $3^{\text {rd }}$ ed., pp.137-166). New York: Wiley.

VanDerHayden, A. M., \& Burns, M. (2013). Universal screening may not be for everyone: Using a threshold model as a smarter way to determine risk. School Psychology Review, 42(4), 402-414.

VanDerHeyden, A. M., \& Witt, J. C. (2005). Quantifying the context of assessment: Capturing the effect of base rates on teacher referral and a problem-solving model of identification. School Psychology Review, 34, 161-183.

Vanderwood, M. L., \& Burns, M. (2013). Validity and school psychology assessment research: Introduction to the special topic. School Psychology Review, 42(4), 363-366.

Vaughn, S., \& Fletcher, J. M. (2010). Thoughts on rethinking response to intervention with secondary students. School Psychology Review, 39(2), 296-299.

Ysseldyke, J. E. (1973). Diagnostic-prescriptive teaching: The search for aptitude-treatment interactions. In L. Mann, \& D. Sabatino (Eds.), The first review of special education. New York: Grune \& Stratton.

Ysseldyke, J. E. (2005). Assessment and decision making for students with learning disabilities: What if this is 
as good as it gets? Learning Disabilities Quarterly, 28(2), 125-128.

Ysseldyke, J., Algozzine, B., \& Epps, S. (1983). A logical and empirical analysis of current practice in classifying students as handicapped. Exceptional Children, 50, 160-165.

Ysseldyke, J., \& Marston, D. (1998). Origins of categorical special education services in schools and a rational for changing them. In D. J. Reschly, W. D. Tilly, \& J. Grimes (Eds.), Functional and non categorical identification and intervention in special education (pp.1-14). Des Moines: Iowa State Department of Education.

Ysseldyke, J., Reynolds, M., \& Weinberg, R. A. (1984). School psychology: A blueprint for training and practice. Minneapolis, M.N: University of Minnesota.

Recebido: julho 31, 2013

Versão final: julho 15, 2014

Aprovado: julho 31, 2014 
